\title{
Bruhat Order on Partial Fixed Point Free Involutions
}

\author{
Mahir Bilen Can* \\ Department of Mathematics \\ Tulane University \\ New Orleans, U.S.A. \\ mcan@tulane.edu \\ Yonah Cherniavsky \\ Department of Computer Science and Mathematics \\ Ariel University \\ Israel \\ yonahch@ariel.ac.il \\ Tim Twelbeck* \\ Department of Mathematics \\ Tulane University \\ New Orleans, U.S.A. \\ ttwelbec@tulane.edu
}

Submitted: May 23, 2014; Accepted: Oct 28, 2014; Published: Nov 13, 2014

Mathematics Subject Classifications: 06A11, 14L30

\begin{abstract}
The order complex of inclusion poset $P F_{n}$ of Borel orbit closures in skewsymmetric matrices is investigated. It is shown that $P F_{n}$ is an EL-shellable poset, and furthermore, its order complex triangulates a ball. The rank-generating function of $P F_{n}$ is computed and the resulting polynomial is contrasted with the Hasse-Weil zeta function of the variety of skew-symmetric matrices over finite fields.
\end{abstract}

Keywords: Bruhat-Chevalley order, partial fixed-point-free involutions, EL-shellability, rank generating function.

\footnotetext{
${ }^{1}$ Supported by the Louisiana Board of Regents Research and Development Grant 549941C1.
} 


\section{Introduction}

This paper is a continuation of our earlier investigations [5], [7] on the Bruhat order on certain sets of involutions, and our notation follows these references closely:

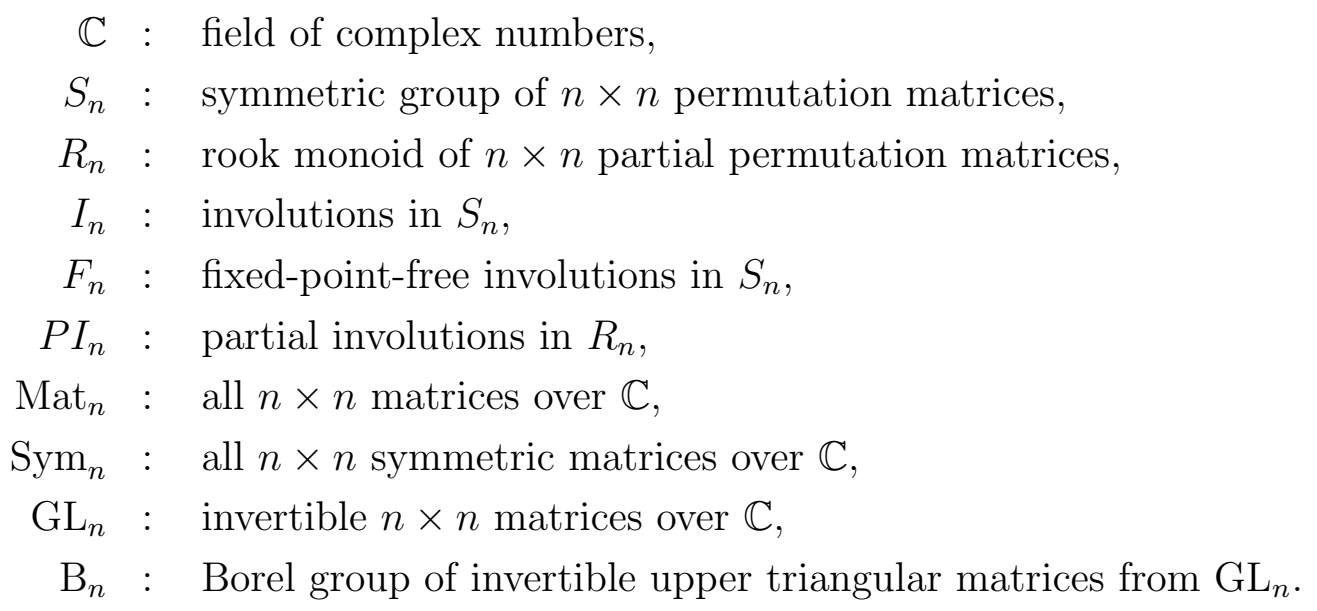

In addition to the above list of notation, we consider $\mathrm{Skew}_{n}$, the space of all $n \times n$ skewsymmetric matrices over $\mathbb{C}$, and $P F_{n}$, the set of all fixed-point-free partial involutions. The purpose of this article is to investigate some combinatorial properties of $P F_{n}$. In some sense, this is the final step of our program for showing that the sets of partial permutations $R_{n}, P I_{n}$, and $P F_{n}$ all share the same algebraic combinatorial properties.

Let $X$ be a variety on which a Borel group $B$ acts algebraically. Let $W$ denote the set of $B$-orbits in $X$, and define the $B$-ordering $\leqslant$ on $W$ by

$$
\mathcal{O}_{1} \leqslant \mathcal{O}_{2} \Longleftrightarrow \mathcal{O}_{1} \subseteq \overline{\mathcal{O}_{2}}, \quad \mathcal{O}_{1}, \mathcal{O}_{2} \in W
$$

Study of this basic combinatorial set-up is important for group theory. Indeed, suppose $G$ is a linear algebraic group with a Borel subgroup $B$. Then the double cosets of $B$ in $G$ are equivalent to the orbits of $B \times B$ acting on $X=G$ via $(g, h) \cdot x=g x h^{-1}$. Furthermore, $B \times B$-orbits in $X$ are parameterized by the 'Weyl group' of $G$ (the Bruhat-Chevalley decomposition). We have a well-known special case, when $G=\mathrm{GL}_{n}$. Then, $\mathrm{B}_{n} \times \mathrm{B}_{n^{-}}$ orbits are parameterized by $S_{n}$, and the induced partial ordering is the Bruhat-Chevalley ordering on $S_{n}$.

In [18], by generalizing Bruhat-Chevalley decomposition to linear algebraic monoids, Renner constructs a rich family of orbit posets. In particular, among other things, he shows that the orbits of the Borel group action

$$
(g, h) \cdot A=g A h^{-1}, g, h \in \mathrm{B}_{n}, A \in \mathrm{Mat}_{n} .
$$

are parameterized by $R_{n}$. Basic combinatorial properties of $\mathrm{B}_{n} \times \mathrm{B}_{n}$-ordering on $R_{n}$ are investigated in [6].

In [19], Richardson and Springer investigate the Borel orbits in the setting of symmetric spaces. In particular, they show that the set of involutions $I_{n}$ of $S_{n}$ parameterizes the Borel 
orbits in the symmetric space $\mathrm{SL}_{n} / \mathrm{SO}_{n}$, and furthermore, the corresponding $\mathrm{B}_{n}$-ordering on $I_{n}$ agrees with the restriction of the Bruhat-Chevalley ordering from $S_{n}$ (see [20]). Here $\mathrm{SL}_{n}$ is the special linear group and $\mathrm{SO}_{n}$ is its special orthogonal subgroup. Also in [19], they show that $\mathrm{B}_{n}$-orbits in $\mathrm{SL}_{n} / \mathrm{Sp}_{n}$ are parameterized by $F_{n} \subset I_{n}$.

The monoid of matrices $\mathrm{Mat}_{n}$ can be viewed as a partial compactification of $\mathrm{GL}_{n}$, and similarly, the set of all symmetric matrices (respectively, set of all skew-symmetric matrices) can be viewed as a partial compactification of $\mathrm{SL}_{n} / \mathrm{SO}_{n}$ (respectively, of $\mathrm{SL}_{2 n} / \mathrm{Sp}_{2 n}$ ). Similar to the construction of $R_{n}$, by using suitable modifications of the method of GaussJordan elimination, it is shown in [22] for $X=\mathrm{Sym}_{n}$, and in [8] for $X=\mathrm{Skew}_{n}$ that the $\mathrm{B}_{n}$-orbits of the action

$$
g \cdot A=\left(g^{-1}\right)^{\top} A g^{-1}, g \in \mathrm{B}_{n}, A \in X
$$

are parameterized by $P I_{n}$ and $P F_{n}$, respectively. Further combinatorial properties of the $\mathrm{B}_{n}$-ordering on $P I_{n}$ and on $P F_{n}$ are investigated by the second author in the papers [1] (joint with E. Bagno) and [8].

There is an interesting relation between $P F_{n}$ and the set of invertible involutions: Let $x \in P F_{n}$ be a partial fixed-point-free involution with determinant 0 . We denote by $\tilde{x}$ the completion of $x$ to an involution in $I_{n}$ by adding the missing diagonal entries. For example,

$$
x=\left(\begin{array}{llll}
0 & 0 & 1 & 0 \\
0 & 0 & 0 & 0 \\
1 & 0 & 0 & 0 \\
0 & 0 & 0 & 0
\end{array}\right) \rightsquigarrow \tilde{x}=\left(\begin{array}{llll}
0 & 0 & 1 & 0 \\
0 & 1 & 0 & 0 \\
1 & 0 & 0 & 0 \\
0 & 0 & 0 & 1
\end{array}\right) .
$$

Define $\phi: P F_{n} \rightarrow I_{n}$ by setting

$$
\phi(x)= \begin{cases}\tilde{x} & \text { if } x \in P F_{n} \backslash F_{n} \\ x & \text { otherwise }\end{cases}
$$

It is not difficult to check that $\phi$ is a bijection between $P F_{n}$ and $I_{n}$ such that $\phi(x)=x$ for all $x \in F_{n}$. Now that we have two sets in bijection with corresponding $\mathrm{B}_{n}$-orderings, it is natural to ask for their comparison. This is one of the goals of our paper.

Recall that the order complex $\Delta(P)$ of a poset $P$ is the abstract simplicial complex consisting of all chains in $P$. Important topological information on a simplicial complex is hidden in the orderings of its facets (which corresponds to the maximal chains in $P$ ). If the facets are ordered in a way that the intersection of a facet with all the preceding facets is a simplicial subcomplex of codimension 1 , then the complex is called shellable. In this case, it is known that the simplicial complex has the homology type of a sphere, or of a ball. For posets, a purely combinatorial criteria for checking the shellability condition is found by Björner in [2], and it is called the "lexicographic shellability" of $P$.

A finite graded poset $P$ with the unique maximum $\hat{1}$ and the unique minimum $\hat{0}$ is called EL-shellable, if there exists a map $f=f_{\Gamma}: C(P) \rightarrow \Gamma$ from the set of covering relations $C(P)$ of $P$ into a totally ordered set $\Gamma$ satisfying 
1. in every interval $[x, y] \subseteq P$ of length $k>0$ there exists a unique saturated chain

$$
\mathfrak{c}: x_{0}=x<x_{1}<\cdots<x_{k-1}<x_{k}=y
$$

such that the entries of the sequence

$$
f(\mathfrak{c})=\left(f\left(x_{0}, x_{1}\right), f\left(x_{1}, x_{2}\right), \ldots, f\left(x_{k-1}, x_{k}\right)\right)
$$

are weakly increasing.

2. The sequence (5) is lexicographically smallest among all sequences of the form

$$
\left(f\left(x_{0}, x_{1}^{\prime}\right), f\left(x_{1}^{\prime}, x_{2}^{\prime}\right), \ldots, f\left(x_{k-1}^{\prime}, x_{k}\right)\right),
$$

where $x_{0}<x_{1}^{\prime}<\cdots<x_{k-1}^{\prime}<x_{k}$.

In literature there are different versions of lexicographic shellability and EL-shellability is known to imply the others (see [23]). A brief history of the shellability questions in Borel orbit posets is as follows: In [11], Edelman proves that BC-order on $S_{n}$ is EL-shellable. Shortly after, Proctor in [16] shows that all classical Weyl groups are EL-shellable. Around the same time, in [3], Björner and Wachs show that Bruchat-Chevalley ordering on all Coxeter groups, as well as on all sets of minimal-length coset representatives (quotients) in Coxeter groups are "dual CL-shellable" (a weaker alternative to EL-shellability). A decade after the introduction of CL-shellability, in [10], M. Dyer shows that Bruhat-Chevalley ordering on all Coxeter groups and all quotients are EL-shellable. As an application of EL-shellability, using Dyer's methods, in [24], L. Williams shows that the poset of cells of a cell decomposition for totally non-negative part of a flag variety is EL-shellable. In the papers [13] and [12] A. Hultman, although avoids showing lex. shellability, obtains the same topological consequences for the Bruhat-Chevalley ordering on "twisted involutions" in Coxeter groups.

There are various directions that the results of [3] are extended. For semigroups, in [17], Putcha shows that "J-classes in Renner monoids" are CL-shellable. In [4], the first author shows that for the special Renner monoid $R_{n}$, not only the $J$-classes are lex. shellable, but also the whole rook monoid $R_{n}$ is EL-shellable. In [7], the first and the third authors show that $P I_{n}$ is EL-shellable. In [5], we show that $F_{n}$ is also EL-shellable, and furthermore, its order complex is a ball of appropriate dimension. In [14], Incitti shows that $I_{n}$ is EL-shellable, and in [15] he shows that the $B$-order on involutions in all classical Weyl groups are EL-shellable.

Contributing to the above literature, we show in this paper that $P F_{n}$ is an EL-shellable poset. Moreover, we show that the order complex of $P F_{n}$ triangulates a ball of dimension $n(n-1) / 2$. On the other hand, it is known that the order complex of $I_{n}$ triangulates a sphere of dimension $\left\lfloor n^{2} / 4\right\rfloor$ (see [14], page 255).

The structure of our paper is as follows. In the next section we introduce basic notation for poset theory. In particular, we recollect some known, basic facts about BruhatChevalley ordering on rooks and partial involutions. In Subsection 2.3, we compare the length functions of $P F_{n}$ and $P I_{n}$. 
Unfortunately, $P F_{n}$ is not a connected subposet of $P I_{n}$, hence we are not able to directly utilize our earlier results from [7]. Therefore, we devote all of Section 3 for the review of the covering relations of $I_{n}, F_{n}$, and of $P I_{n}$ in order for describing the covering relations of $P F_{n}$ next.

In Section 4 we present our proof of EL-shellability of $P F_{n}$. As an application of this result, in Section 5, we determine the homotopy type of the order complex of the proper part of $P F_{n}$, namely $P F_{n}$ with its smallest and the largest elements excluded.

In the final section of our paper, we investigate the length-generating functions of certain subposets of $P F_{n}$. In particular, we relate our length generating function computations to the number of rational points of the variety of skew-symmetric matrices of fixed rank defined over a finite field.

\section{Preliminaries}

Notation: Let $m$ be a positive integer. We denote the set $\{1, \ldots, m\}$ by $[m]$. The rank of a matrix $x \in \mathrm{Mat}_{n}$ is denoted by $r k(x)$.

\subsection{Poset terminology}

All of our posets are assumed to be finite, graded, and furthermore, they are assumed to possess a minimal and a maximal element, denoted by $\hat{0}$ and $\hat{1}$, respectively. We reserve the letter $P$ as the name of a generic such poset and denote by $\ell: P \rightarrow \mathbb{N}$ (or, by $\ell_{P}$, if needed) the length function on $P$. The set of all covering relations in $P$ is denoted by $C(P)$. If $(x, y) \in C(P)$, then we write $y \rightarrow x$ to mean that $y$ covers $x$.

Recall that the Möbius function of $P$ is defined recursively by the formula

$$
\begin{aligned}
& \mu([x, x])=1, \\
& \mu([x, y])=-\sum_{x \leqslant z<y} \mu([x, z])
\end{aligned}
$$

for all $x \leqslant y$ in $P$. As customary, we denote by $\Delta(P)$ the order complex of $P$. It is well known that $\mu(\hat{0}, \hat{1})$ is equal to the "reduced Euler characteristic" $\tilde{\chi}(\Delta(P))$ of the topological realization of $\Delta(P)$. See Proposition 3.8.6 in [21].

Let $\Gamma$ denote a finite totally ordered set and let $g$ be a $\Gamma$-valued function defined on $C(P)$. Then $g$ is called an $R$-labeling of $P$, if for every interval $[x, y]$ in $P$, there exists a unique chain $x=x_{1} \leftarrow x_{2} \leftarrow \cdots \leftarrow x_{n-1} \leftarrow x_{n}=y$ such that

$$
g\left(x_{1}, x_{2}\right) \leqslant g\left(x_{2}, x_{3}\right) \leqslant \cdots \leqslant g\left(x_{n-1}, x_{n}\right) .
$$

Thus, $P$ is EL-shellable, if it has an $R$-labeling $g: C(P) \rightarrow \Gamma$ such that for each interval $[x, y]$ in $P$ the sequence (6) is lexicographically smallest among all sequences of the form

$$
\left(g\left(x, x_{2}^{\prime}\right), g\left(x_{2}^{\prime}, x_{3}^{\prime}\right), \ldots, g\left(x_{k-1}^{\prime}, y\right)\right)
$$


where $x \leftarrow x_{2} \leftarrow^{\prime} \cdots \leftarrow x_{k-1}^{\prime} \leftarrow y$.

For $S \subseteq[n]$, by $P_{S}$ we denote the subset $P_{S}=\{x \in P: \ell(x) \in S\}$, and denote by $\mu_{S}$ the Möbius function of the poset $\hat{P}_{S}$ that is obtained from $P_{S}$ by adjoining a smallest and a largest element, if they are missing. For an $R$-labeling $g: C(P) \rightarrow \Gamma$ of $P$, it is well known that the quantity $(-1)^{|S|-1} \mu_{S}\left(\hat{0}_{\hat{P}_{S}}, \hat{1}_{\hat{P}_{S}}\right)$ is equal to the number of maximal chains $x_{0}=\hat{0} \leftarrow x_{1} \leftarrow \cdots \leftarrow x_{n}=\hat{1}$ in $P$ for which the sequence $\left(g\left(x_{0}, x_{1}\right), \cdots, g\left(x_{n-1}, x_{n}\right)\right.$ has descent set $S$, that is to say, for which $\left\{i \in[n]: g\left(x_{i-1}, x_{i}\right) \geqslant g\left(x_{i+1}, x_{i}\right)\right\}=S$. See Theorem 3.14 .2 in [21].

\section{$2.2 \quad B$-order on partial involutions}

The notation $F_{n}, I_{n}, P I_{n}, R_{n}, S_{n}$, Skew $n$, and $\mathrm{Sym}_{n}$ are as in the introduction.

Recall that $R_{n}$ parameterizes the $\mathrm{B}_{n} \times \mathrm{B}_{n}$-orbits in Mat ${ }_{n}$. For the purposes of this paper, it is more natural for us to look at the inclusion poset of $\mathrm{B}_{n}^{\top} \times \mathrm{B}_{n}$-orbit closures in $R_{n}$, whose dual poset we denote by $\left(R_{n}, \leqslant_{R o o k}\right)$. Here $\mathrm{B}_{n}^{\top}$ is the Borel subgroup of all lower triangular matrices from $\mathrm{GL}_{n}$.

In [8], the second author shows that the Borel orbits in $\mathrm{Skew}_{n}$ are parameterized by those elements $x \in \mathrm{Skew}_{n}$ such that

1. the entries of $x$ are either 0,1 or -1 ,

2. any non-zero entry of $x$ that is above the main diagonal is a +1 ,

3. in every row and column of $x$ there exists at most one non-zero entry.

Note that when -1 's in $x$ are replaced by +1 's, the resulting matrix $\tilde{x}$ is a partial involution with no diagonal entry. In other words, $\tilde{x}$ is a fixed-point-free partial involution. It is obvious that this correspondence is a bijection, hence $P F_{n}$ parameterizes the Borel orbits in Skew $_{n}$.

Containment relations among the closures of Borel orbits in Skew ${ }_{n}$ define a partial ordering on $P F_{n}$. We denote its dual by $\leqslant_{S k e w}$. Similarly, on $P I_{n}$ we have the dual of the partial ordering induced from the containment relations among the Borel orbit closures in $\operatorname{Sym}_{n}$. We denote this dual partial ordering by $\leqslant_{\text {Sym }}$.

\subsection{Combinatorial approach to the posets $R_{n}, P I_{n}, P F_{n}$}

There is a combinatorial method for deciding when two elements $x$ and $y$ from $\left(R_{n}, \leqslant_{\text {Rook }}\right)$ (respectively, from $\left(P I_{n}, \leqslant_{\text {Sym }}\right)$, or from $\left(P F_{n}, \leqslant_{\text {Skew }}\right)$ ) are comparable with respect to $\leqslant_{\text {Rook }}$ (respectively, with respect to $\leqslant_{S y m}$, or $\leqslant_{\text {Skew }}$ ). We denote by $R k(x)$ the matrix whose $i, j$-th entry is the rank of the upper left $i \times j$ submatrix of $x$. Hence, $R k(x)$ is an $n \times n$ matrix with non-negative integer entries. We call $R k(x)$, the rank-control matrix of $x$.

Let $A=\left(a_{i, j}\right)$ and $B=\left(b_{i, j}\right)$ be two matrices of the same size with real number entries. We write $A \leqslant B$ if $a_{i, j} \leqslant b_{i, j}$ for all $i$ and $j$. Then

$$
x \leqslant \text { Rook } y \Longleftrightarrow R k(y) \leqslant R k(x) \text {. }
$$


The same criterion holds for the posets $\leqslant_{\text {Sym }}$ and $\leqslant_{\text {Skew }}$.

We recall some fundamental facts about the covering relations of $\leqslant_{\text {Sym }}$ and $\leqslant_{\text {Skew }}$. Our references are [1] and [8]. Let $R k(x)=\left(r_{i, j}\right)_{i, j=1}^{m}$ denote the rank-control matrix of an $m \times m$ matrix $x$. As a notation we set $r_{0, i}=0$ for $i=0, \ldots, m$ and define

$$
\begin{aligned}
& \rho_{\leqslant}(x)=\#\left\{(i, j): 1 \leqslant i \leqslant j \leqslant n \text { and } r_{i, j}=r_{i-1, j-1}\right\}, \\
& \rho_{<}(x)=\#\left\{(i, j): 1 \leqslant i<j \leqslant n \text { and } r_{i, j}=r_{i-1, j-1}\right\} .
\end{aligned}
$$

Then the length function $\ell_{P F_{n}}$ of the poset $P F_{n}$ is equal to the restriction of $\rho_{<}$to $P F_{n}$. Furthermore, $x$ covers $y$ if and only if $R k(x) \leqslant R k(y)$ and $\ell_{P F_{n}}(x)-\ell_{P F_{n}}(y)=1$.

The length function of $P F_{n}$ differs from the length function of $P I_{n}$ in two ways: The ranks of two matrices $y<x$ in $P F_{n}$ differ by a multiple of 2 , and the smallest element in $P I_{n}$ is the identity matrix, which is not in $P F_{n}$. The minimal element in $P F_{n}$ is given by the matrix with the largest rank-control matrix. This means that in the case when $n$ is even $\ell_{P F_{n}}(x)=\ell_{P I_{n}}(x)-\frac{n-r k(x)}{2}-\frac{n}{2}$. We subtract $\frac{n-r k(x)}{2}$ so that the length function increases only by 1 if the rank drops by 2 and we subtract $\frac{n}{2}$ because the minimal element has to have length zero. Similarly, when $n$ is odd we have to subtract $\frac{n-1-r k(x)}{2}$ and $\frac{n+1}{2}$. Summarizing, we see that for all $n$ the length function $\ell_{P F_{n}}(x)$ of $P F_{n}$ is given by

$$
\begin{aligned}
\ell_{P F_{n}}(x) & =\ell_{P I_{n}}(x)-\frac{n-r k(x)}{2}-\frac{n}{2} \\
& =\ell_{P I_{n}}(x)-\frac{2 n-r k(x)}{2} \\
& =\rho_{<}(x)-\frac{2 n-r k(x)}{2} .
\end{aligned}
$$

Example 1. When $n=6$, the smallest element is

$$
\omega_{0}=\left(\begin{array}{llllll}
0 & 1 & 0 & 0 & 0 & 0 \\
1 & 0 & 0 & 0 & 0 & 0 \\
0 & 0 & 0 & 1 & 0 & 0 \\
0 & 0 & 1 & 0 & 0 & 0 \\
0 & 0 & 0 & 0 & 0 & 1 \\
0 & 0 & 0 & 0 & 1 & 0
\end{array}\right),
$$

and when $n=5$, the smallest element is $\omega_{0}=\left(\begin{array}{ccccc}0 & 1 & 0 & 0 & 0 \\ 1 & 0 & 0 & 0 & 0 \\ 0 & 0 & 0 & 1 & 0 \\ 0 & 0 & 1 & 0 & 0 \\ 0 & 0 & 0 & 0 & 0\end{array}\right)$.

\section{An EL-labeling of $P I_{n}$}

We recall some results on the covering relations of $I_{n}, F_{n}$, and of $P I_{n}[14,5,7]$. 


\subsection{EL-labeling of $\boldsymbol{I}_{n}$}

For a permutation $\sigma \in S_{n}$, a rise of $\sigma$ is a pair of indices $1 \leqslant i_{1}, i_{2} \leqslant n$ such that

$$
i_{1}<i_{2} \text { and } \sigma\left(i_{1}\right)<\sigma\left(i_{2}\right) .
$$

A rise $\left(i_{1}, i_{2}\right)$ is called free, if there is no $k \in[n]$ such that

$$
i_{1}<k<i_{2} \text { and } \sigma\left(i_{1}\right)<\sigma(k)<\sigma\left(i_{2}\right) .
$$

For $\sigma \in S_{n}$, define its fixed point set, its exceedance set and its defect set to be

$$
\begin{aligned}
& I_{f}(\sigma)=\operatorname{Fix}(\sigma)=\{i \in[n]: \sigma(i)=i\}, \\
& I_{e}(\sigma)=\operatorname{Exc}(\sigma)=\{i \in[n]: \sigma(i)>i\}, \\
& I_{d}(\sigma)=\operatorname{Def}(\sigma)=\{i \in[n]: \sigma(i)<i\},
\end{aligned}
$$

respectively.

Given a rise $\left(i_{1}, i_{2}\right)$ of $\sigma$, its type is defined to be the pair $(a, b)$, if $i_{1} \in I_{a}(\sigma)$ and $i_{2} \in I_{b}(\sigma)$, for some $a, b \in\{f, e, d\}$. We call a rise of type $(a, b)$ an $a b$-rise. On the other hand, two kinds of ee-rises have to be distinguished from each other; an ee-rise is called crossing, if $i_{1}<\sigma\left(i_{1}\right)<i_{2}<\sigma\left(i_{2}\right)$, and it is called non-crossing, if $i_{1}<i_{2}<\sigma\left(i_{1}\right)<\sigma\left(i_{2}\right)$.

The rise $\left(i_{1}, i_{2}\right)$ of an involution $\sigma \in I_{n}$ is called suitable if it is free and if its type is one of the following: $(f, f),(f, e),(e, f),(e, e),(e, d)$.

A covering transformation, denoted $c t_{\left(i_{1}, i_{2}\right)}(\sigma)$, of a suitable rise $\left(i_{1}, i_{2}\right)$ of $\sigma$ is the involution obtained from $\sigma$ by moving the 1's from the black dots to the white dots as depicted in Figure 1.

It is shown in [14] that if $\tau$ and $\sigma$ are two involutions in $I_{n}$, then

$\tau$ covers $\sigma$ in $\leqslant_{S y m} \Longleftrightarrow \tau=c t_{\left(i_{1}, i_{2}\right)}(\sigma)$, for some suitable rise $\left(i_{1}, i_{2}\right)$ of $\sigma$.

Let $\Gamma$ denote the totally ordered set $[n] \times[n]$ with respect to lexicographic ordering. In the same paper, Incitti shows that the labeling defined by

$$
f_{\Gamma}\left(\left(\sigma, c t_{\left(i_{1}, i_{2}\right)}(\sigma)\right)\right):=\left(i_{1}, i_{2}\right) \in \Gamma
$$

is an EL-labeling, hence, $\left(I_{n}, \leqslant_{S y m}\right)$ is an EL-shellable poset.

\subsection{EL-labeling of $F_{2 n}$}

Recall that $F_{2 n}$ is a connected graded subposet of $I_{2 n}$. Therefore, its covering relations are among the covering relations of $I_{2 n}$. On the other hand, within $F_{2 n}$ we use two types of covering transformations, only: a non-crossing ee-rise and an ed-rise. These moves correspond to the items numbered 4 and 6 in Table 1 of [14]. It is shown in [5] that these covering labels is an EL-labeling for $F_{2 n}$. 

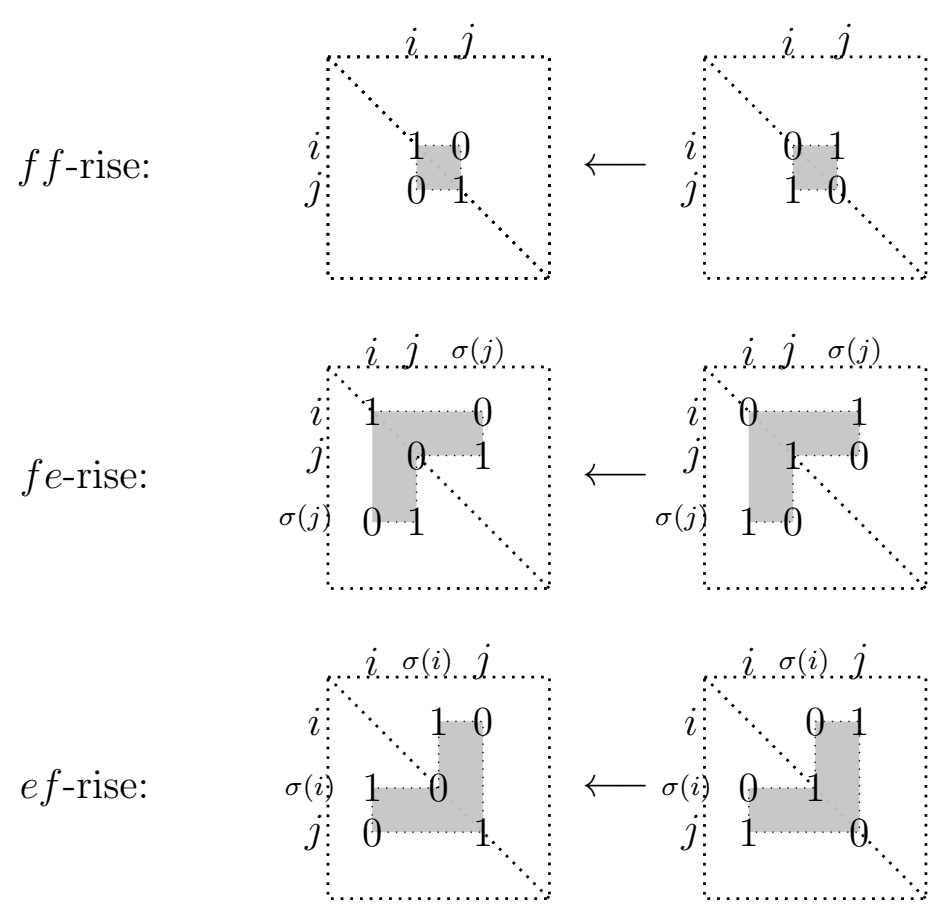

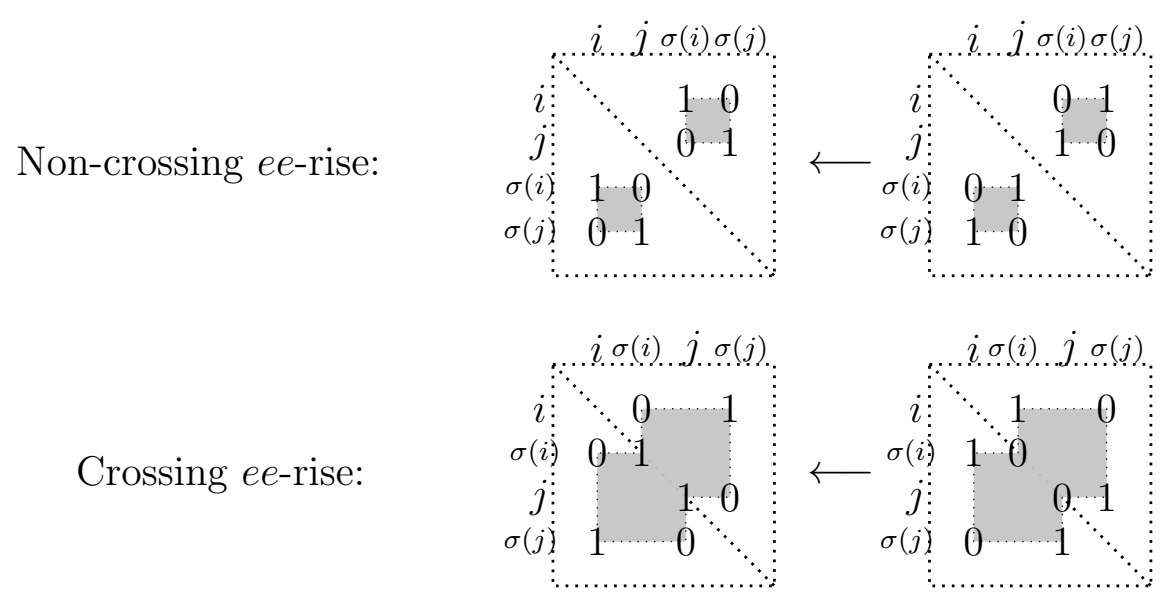

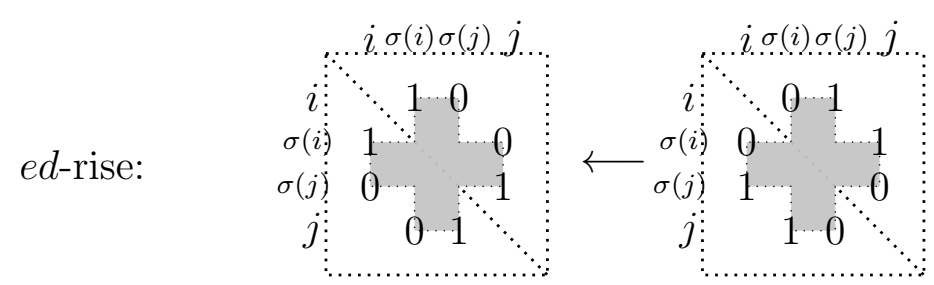

Figure 1: Covering transformations $\sigma \leftarrow \tau=c t_{(i, j)}(\sigma)$ of $I_{n}$. 


\subsection{EL-labeling of $P I_{n}$}

When two partial involutions $x$ and $y$ have the same zero rows and zero columns, the covering relation $x \rightarrow y$ is the same as in the case of invertible involutions.

\section{Example 2.}

$$
y=\left(\begin{array}{lll}
1 & 0 & 0 \\
0 & 0 & 0 \\
0 & 0 & 1
\end{array}\right) \text { is covered by } x=\left(\begin{array}{lll}
0 & 0 & 1 \\
0 & 0 & 0 \\
1 & 0 & 0
\end{array}\right)
$$

Note that $x \rightarrow y$ if and only if the invertible involution $\tilde{x}$, that is obtained from $x$ by removing the rows and columns of $x$ with no non-zero entries, covers the invertible involution $\tilde{y}$ that is obtained from $y$ by removing its rows and columns with zeros only.

Moving down a non-zero entry along the diagonal gives a covering relation:

\section{Example 3.}

$$
y=\left(\begin{array}{llll}
0 & 0 & 1 & 0 \\
0 & 1 & 0 & 0 \\
1 & 0 & 0 & 0 \\
0 & 0 & 0 & 0
\end{array}\right) \text { is covered by } x=\left(\begin{array}{llll}
0 & 0 & 1 & 0 \\
0 & 0 & 0 & 0 \\
1 & 0 & 0 & 0 \\
0 & 0 & 0 & 1
\end{array}\right)
$$

Similarly,

$$
y=\left(\begin{array}{lll}
0 & 0 & 0 \\
0 & 1 & 0 \\
0 & 0 & 1
\end{array}\right) \text { is covered by } x=\left(\begin{array}{lll}
0 & 0 & 0 \\
0 & 1 & 0 \\
0 & 0 & 0
\end{array}\right)
$$

Another type of covering relation is obtained by the moving of off-diagonal pairs $(i, j)$ and $(j, i)$, where $i>j$ to down/right, or to right/down available positions.

Example 4. There are two cases:

$$
\begin{aligned}
& \text { 1. } y=\left(\begin{array}{lll}
0 & 1 & 0 \\
1 & 0 & 0 \\
0 & 0 & 0
\end{array}\right) \text { is covered by } x=\left(\begin{array}{lll}
0 & 0 & 1 \\
0 & 0 & 0 \\
1 & 0 & 0
\end{array}\right) \text {, } \\
& \text { 2. } y=\left(\begin{array}{lll}
0 & 0 & 1 \\
0 & 0 & 0 \\
1 & 0 & 0
\end{array}\right) \text { is covered by } x=\left(\begin{array}{lll}
0 & 0 & 0 \\
0 & 0 & 1 \\
0 & 1 & 0
\end{array}\right) \text {. }
\end{aligned}
$$

When a down/right move is performed on $y$ (as in part 2. of Example 4), there may not be any available positions to place the non-zero entries of $x$. In this case, the pushed entries are placed on the diagonal. If there are no available diagonal entries for both of the 1's, then one of them is pushed out of the matrix. 
Example 5. Once again, there are two moves of similar nature:

1. $y=\left(\begin{array}{llll}0 & 0 & 1 & 0 \\ 0 & 1 & 0 & 0 \\ 1 & 0 & 0 & 0 \\ 0 & 0 & 0 & 0\end{array}\right)$ is covered by $x=\left(\begin{array}{llll}0 & 0 & 0 & 0 \\ 0 & 1 & 0 & 0 \\ 0 & 0 & 1 & 0 \\ 0 & 0 & 0 & 1\end{array}\right)$.
2. $y=\left(\begin{array}{ll}0 & 1 \\ 1 & 0\end{array}\right)$ is covered by $x=\left(\begin{array}{ll}0 & 0 \\ 0 & 1\end{array}\right)$.

In the light of the above examples, we label a covering relation $x \rightarrow y$ in $P I_{n}$ as follows.

Definition 6. 1. As in Example 2, if the covering relation $x \rightarrow y$ is derived from the covering relation $\tilde{x} \rightarrow \tilde{y}$ of invertible involutions that are obtained from $x$ and $y$, respectively, then we use the labeling $\tilde{x} \rightarrow \tilde{y}$ as defined in [14].

2. If the covering relation results from a move as in Example 3, namely from a diagonal push where the element that is pushed from is at the position $(i, i)$, then we label it by $(i, i)$.

3. Suppose $x \rightarrow y$ is as in Example 4, or 5. Observe that, in all of these covering relations, one of the 1's is pushed down and the other is pushed right. Let $i$ denote the column index of the first 1 that is pushed to the right, and let $j$ denote the index of the resulting column. Then we label the covering by $(i, j)$.

To illustrate the third labeling let us present a few more examples.

\section{Example 7.}

$$
y=\left(\begin{array}{lllll}
0 & 0 & 0 & 1 & 0 \\
0 & 0 & 1 & 0 & 0 \\
0 & 1 & 0 & 0 & 0 \\
1 & 0 & 0 & 0 & 0 \\
0 & 0 & 0 & 0 & 0
\end{array}\right) \text { is covered by } x=\left(\begin{array}{lllll}
0 & 0 & 0 & 1 & 0 \\
0 & 0 & 0 & 0 & 1 \\
0 & 0 & 0 & 0 & 0 \\
1 & 0 & 0 & 0 & 0 \\
0 & 1 & 0 & 0 & 0
\end{array}\right)
$$

The corresponding labeling here is $(3,5)$.

\section{Example 8.}

$$
y=\left(\begin{array}{llllll}
0 & 0 & 0 & 0 & 0 & 1 \\
0 & 0 & 0 & 0 & 1 & 0 \\
0 & 0 & 0 & 0 & 0 & 0 \\
0 & 0 & 0 & 0 & 0 & 0 \\
0 & 1 & 0 & 0 & 0 & 0 \\
1 & 0 & 0 & 0 & 0 & 0
\end{array}\right) \text { is covered by } x=\left(\begin{array}{cccccc}
0 & 0 & 0 & 0 & 0 & 0 \\
0 & 0 & 0 & 0 & 1 & 0 \\
0 & 0 & 0 & 0 & 0 & 1 \\
0 & 0 & 0 & 0 & 0 & 0 \\
0 & 1 & 0 & 0 & 0 & 0 \\
0 & 0 & 1 & 0 & 0 & 0
\end{array}\right)
$$

The corresponding labeling here is $(1,3)$. 


\section{Example 9.}

$$
y=\left(\begin{array}{lllll}
0 & 0 & 0 & 1 & 0 \\
0 & 0 & 1 & 0 & 0 \\
0 & 1 & 0 & 0 & 0 \\
1 & 0 & 0 & 0 & 0 \\
0 & 0 & 0 & 0 & 0
\end{array}\right) \text { is covered by } x=\left(\begin{array}{lllll}
0 & 0 & 0 & 1 & 0 \\
0 & 0 & 0 & 0 & 0 \\
0 & 0 & 1 & 0 & 0 \\
1 & 0 & 0 & 0 & 0 \\
0 & 0 & 0 & 0 & 1
\end{array}\right)
$$

The corresponding labeling here is $(2,3)$.

Definition 10. If $x$ covers $y$ with label $(i, j)$, then we refer to it as an $(i, j)$-covering and say that $y$ is obtained from $x$ by an $(i, j)$-move. More briefly, we call a covering relation a $c$-cover, if it is derived from an involution; a $d$-cover, if it is obtained by a shift of a diagonal element; an $r$-cover, if it is derived from a right/down, or from a down/right move. The corresponding moves of 1's are referred to as $c$-, $d$ - and $r$-moves.

Lemma 11 (Lemma 16, [7]). Let $x$ and $y$ be two partial involutions. Then $x$ covers $y$ if and only if one of the following is true:

1. $x$ is obtained from $y$ by a c-move as in Example 2.

2. Without removing a suitable rise, $x$ is obtained from $y$ by one of the following moves:

(a) a d-move, as in Example 3,

(b) an r-move, as in Example 4, or as in Example 5.

It is shown in [7] that the covering labelings defined in Definition 10 is an EL-labeling for $P I_{n}$.

\section{An EL-labeling of $\boldsymbol{P} \boldsymbol{F}_{n}$}

Covering relations of $F_{n}$ are covering relations in $I_{n}$, as well. Unfortunately, this is not the case for $P F_{n}$ relative to $P I_{n}$. In other words, as a subposet of $P I_{n}, P F_{n}$ is not connected. For example, when $n=2$, there are only two partial fixed-point-free involutions: $x=\left(\begin{array}{ll}0 & 0 \\ 0 & 0\end{array}\right)$ and $y=\left(\begin{array}{ll}0 & 1 \\ 1 & 0\end{array}\right)$, hence $x$ covers $y$ as a partial fixed-point-free involution. However, viewed as a partial involution $x$ does not cover $y$ since $y<\left(\begin{array}{ll}0 & 0 \\ 0 & 1\end{array}\right)<x$.

Lemma 12. Suppose $x \rightarrow y$ in $P F_{n}$. Then either $x$ covers $y$ as an element of $P I_{n}$, or there exists $z \in P I_{n}$ such that $x \rightarrow z$ by an $d$-cover as an element of $P I_{n}$, and $z \rightarrow y$ by an $r$-cover in $P I_{n}$, where at each step the rank drops by 1. Furthermore, in the first case, there are two possibilities:

1. $x \rightarrow y$ is an $r$-cover in $P I_{n}$, or 
2. $x \rightarrow y$ is a c-cover corresponding to a non-crossing ee, or to an ed-rise in $P I_{n}$.

Proof. Obviously, if $x$ covers $y$ in $P I_{n}$ and if both $x$ and $y$ are members of $P F_{n}$, then $x$ covers $y$ in $P F_{n}$, also. Thus, the last assertion follows from Lemma 11.

We proceed with the assumption that $x, y \in P F_{n}$ but $x$ does not cover $y$ in $P I_{n}$. Towards a contradiction, assume that there does not exists $z \in P I_{n}$ as in the conclusion of the lemma. This means that the open interval $(y, x)=\left\{z \in P I_{n}: y<z<x\right\}$ lies in $P I_{n} \backslash P F_{n}$. In other words, any $z \in(y, x)$ has to have a non-zero diagonal entry. This eliminates the possibility of $z \rightarrow y$ being a $c$-cover (see Figure 1). Clearly, $z \rightarrow y$ cannot be a $d$-cover, neither.

We continue with the assumption that $z$ is obtained from $y$ by an $r$-move, which places two symmetric entries on the diagonal. In this case, another $r$-move is possible in $y$ involving the same 1's. (To construct an example to this situation, start with $y$ as in Example 9.) Let $z_{1}$ denote this new element from $P F_{n}$. By comparing their rank-control matrices, we see that $R k(x)<R k\left(z_{1}\right)$, hence $y<z_{1}<x$. This contradicts with our assumption that the interval $(y, x)$ lies in $P I_{n} \backslash P F_{n}$. Therefore, $z$ covers $y$ by an $r$-move, by deleting a 1 from $y$ and placing another to diagonal. Then by a $d$-move removing this diagonal 1 we obtain $x$. Thus we obtain a contradiction to our initial assumption.

Remark 13. Let $x$ and $y$ be two elements from $P F_{n}$ such that $x$ covers $y$ by an $r$-move. Let $x=\left(x_{1}, \ldots, x_{n}\right)$ and $y=\left(y_{1}, \ldots, y_{n}\right)$ denote $x$ and $y$ in one-line notation. Then exactly one of the following statements is true:

1. $x$ is obtained from $y$ by replacing exactly two entries of $y=\left(y_{1}, \ldots, y_{n}\right)$ by 0 's.

2. There exists $i \in[n]$ such that $x$ is obtained from $y$ by replacing $y_{i}$ by the number $x_{i}$, setting $y_{i}$-th entry of $y$ to 0 and replacing the $x_{i}$-th entry of $y$ (which is a 0 ) by $i$.

In the light of Lemma 12 we give the following definition.

Definition 14. 1. If the covering relation is derived from a $c$-move, then we use the labeling as defined in [7] and transform this label $(i, j)$ into $(n-i, n-j)$.

2. If the covering relation $x \rightarrow y$ results from an $r$-move, then we define the label to be $(i+n, j)$, where $x>y$ results from $y$ by moving the 1 in column $i$ to row $j$. If the 1 is pushed out of the matrix, then we set $j=n+1$.

In the case of invertible fixed-point-free involutions we show in [5] that the lexicographically largest chain is the only decreasing chain. Since the label is transformed from $(i, j)$ to $(n-i, n-j)$ now the lexicographically smallest chain is increasing. The reason the label of $r$-moves is shifted by $n$ in the first coordinate is to ensure that every $r$-cover has a bigger label than any c-cover. In Figure 2, we illustrate Definition 14. 


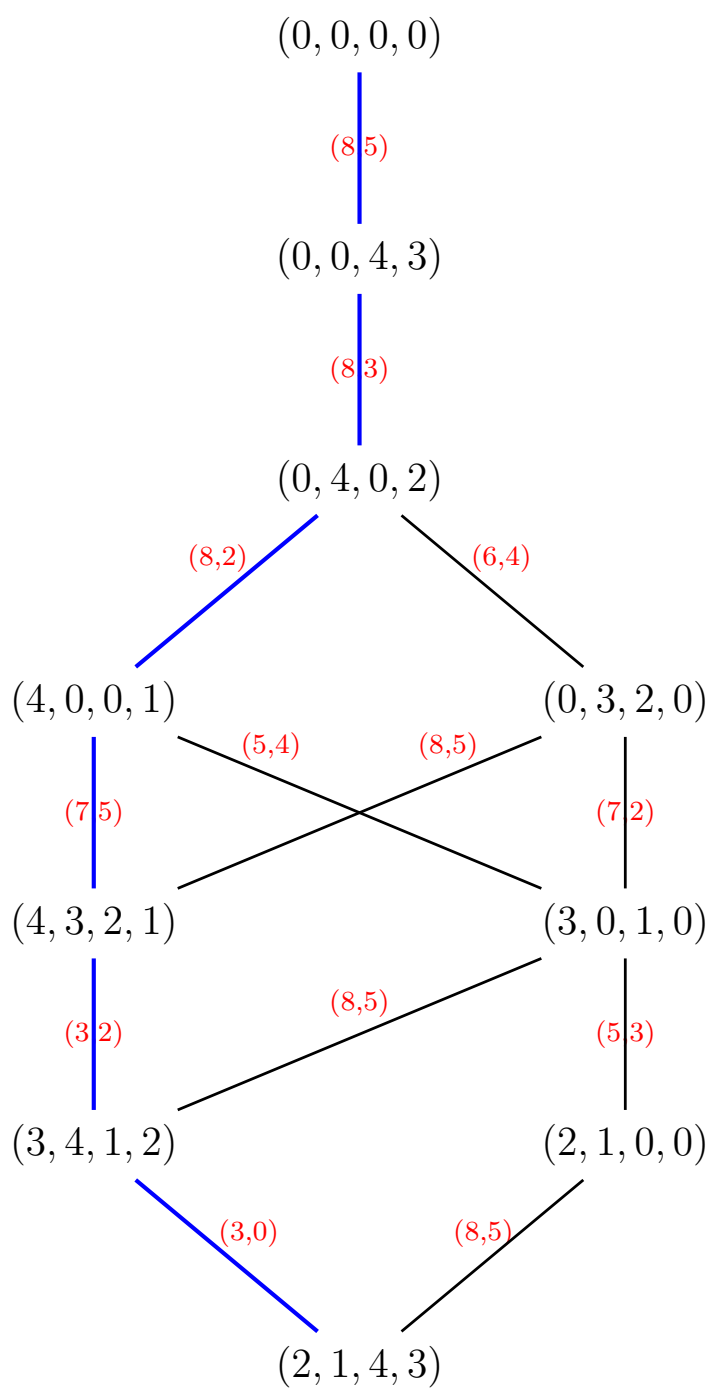

Figure 2: The EL-labeling of $P F_{4}$.

Proposition 15. Let $y<x$ be two partial fixed-point-free involutions from $P F_{n}$, and let

$$
\mathfrak{c}: x=x_{1}<x_{2}<\cdots<x_{s+1}=y
$$

denote the saturated chain whose sequence of labels $f(\mathfrak{c})$, as defined in Definition 14, is lexicographically smallest among all such sequences. Then $f(\mathfrak{c})$ is a weakly increasing sequence.

Proof. Towards a contradiction assume that $f(\mathfrak{c})$ is not weakly increasing. Then there exist three consecutive terms

$$
x_{t-1}<x_{t}<x_{t+1}
$$

in $\mathfrak{c}$ such that $f\left(\left(x_{t-1}, x_{t}\right)\right)>f\left(\left(x_{t}, x_{t+1}\right)\right)$. We have 4 cases to consider: 
Case 1: type $\left(\left(x_{t-1}, x_{t}\right)\right)=c$, and type $\left(\left(x_{t}, x_{t+1}\right)\right)=c$,

Case 2: type $\left(\left(x_{t-1}, x_{t}\right)\right)=r$, and type $\left(\left(x_{t}, x_{t+1}\right)\right)=r$,

Case 3: type $\left(\left(x_{t-1}, x_{t}\right)\right)=c$, and type $\left(\left(x_{t}, x_{t+1}\right)\right)=r$,

Case 4: type $\left(\left(x_{t-1}, x_{t}\right)\right)=r$, and type $\left(\left(x_{t}, x_{t+1}\right)\right)=c$.

In each of these cases, we either produce an immediate contradiction by showing that the two moves are interchangeable (hence $\mathfrak{c}$ is not the smallest chain), or we construct an element $z \in[x, y] \cap P F_{n}$ which covers $x_{t-1}$, and such that $f\left(\left(x_{t-1}, z\right)\right)<f\left(\left(x_{t-1}, x_{t}\right)\right)$. Since we assume that $f(\mathfrak{c})$ is the lexicographically smallest Jordan-Hölder sequence, the existence of such an element $z$ is a contradiction, also.

To this end, suppose that the label of the first move $\left(x_{t} \rightarrow x_{t-1}\right)$ is $(i, j)$, and the second move $\left(x_{t+1} \rightarrow x_{t}\right)$ is labeled by $(k, l)$.

Case 1: Follows from the proof for invertible fixed-point-free involutions, see [5].

Case 2: If $i=k$, then $l>j$. In this case, we interchange the two moves to obtain our desired contradiction. Therefore we continue with assuming $k<i$. If $k-n=j$ then $j<i-n$ and $(m+n, l)$ is possible in $x_{t-1}$ with $m<j<i$, where $(m, i-n)$ is the position of the 1 in $x_{t-1}$. If $k-n \neq j$ then either the two moves are interchangeable, or $(k, l)$ removes a suitable rise in $x_{t-1}$ which corresponds to a move with a smaller label than $(i, j)$.

Case 3: This case is impossible since every $c$-move has a smaller label than any $r$-move.

Case 4: If the $r$-cover labeled $(i, j)$ is the covering relation with the lexicographically smallest label then there is no suitable rise in $x_{t-1}$. The $c$-move has to involve one of the moved 1's since otherwise there is a suitable rise in $x_{t-1}$. For this, one of the moved 1's has to have a 1 to the upper left or the lower right in $x_{t}$ that was not to the upper left or lower right of it in $x_{t-1}$. Since the 1's are moved right and down respectively, it is impossible that there is a 1 to the lower right in $x_{t}$ that is not to the lower right in $x_{t-1}$. If the $c$-cover corresponds to the suitable rise $(m, i-n)$ (with label $(n-m, i))$, then $(i, j)$ is not the $r$-move with the smallest label in $x_{t-1}$ since in this case $(m+n, j)$ is possible in $x_{t-1}$ with $(n+m, j)<(i, j)$. If the $c$-cover corresponds to the rise $(m, j)$, then the $r$-move $(m+n, i-n)$ is possible in $x_{t-1}$ which again has a smaller label than $(i, j)$.

Proposition 16. We retain the notation from (the proof of) Proposition 15. Then $f(\mathfrak{c})$ is the unique increasing chain in $[y, x]$.

Proof. We use induction on the length $s+1$ of the interval $[y, x]$ to prove that no other chain is lexicographically increasing. Clearly, if $x$ covers $y$, there is nothing to prove, so, we assume that for any interval of length $k \leqslant s$ there exists a unique increasing maximal chain.

Assume that there exists another increasing chain

$$
\mathfrak{c}^{\prime}: y=x_{0}<x_{1}^{\prime}<\cdots<x_{s}^{\prime}<x_{s+1}=x .
$$

Since the length of the chain

$$
x_{1}^{\prime}<\cdots<x_{s}^{\prime}<x_{s+1}=x
$$


is $s$, by the induction hypothesis, it is the lexicographically smallest chain between $x_{1}^{\prime}$ and $x$. We are going to find contradictions to each of the following possibilities:

Case 1: type $\left(x_{0}, x_{1}\right)=c$, and type $\left(x_{0}, x_{1}^{\prime}\right)=c$,

Case 2: type $\left(x_{0}, x_{1}\right)=r$, and type $\left(x_{0}, x_{1}^{\prime}\right)=r$,

Case 3: type $\left(x_{0}, x_{1}\right)=c$, and type $\left(x_{0}, x_{1}^{\prime}\right)=r$,

Case 4: type $\left(x_{0}, x_{1}\right)=r$, and type $\left(x_{0}, x_{1}^{\prime}\right)=c$.

In each of these cases we will construct a partial fixed-point-free involution $z \in[y, x]$ such that $z$ covers $x_{1}^{\prime}$ and $f\left(\left(x_{1}^{\prime}, z\right)\right)<f\left(\left(x_{1}^{\prime}, x_{2}^{\prime}\right)\right)$, contradicting the induction hypothesis. To this end, let $f\left(\left(x_{0}, x_{1}\right)\right)=(i, j), f\left(\left(x_{0}, x_{1}^{\prime}\right)\right)=(k, l)$ and assume that $(k, l)<(i, j)$.

Case 1: Done in the proof for the invertible case, see [5].

Case 2: It is impossible for $i=k$ since there is only one $r$-move for each 1 . Therefore assume that $i<k$. Let the moved 1's be on the symmetric positions $(i-n, m)$ and $(m, i-n)$ in $x_{0}$. If $k=m+n$ then $(l+n, j)$ is possible in $x_{1}^{\prime}$ with $(l+n, j)<(k, l)$. If $k \neq m$ then either the two moves are interchangeable or the suitable rise $(n-i, n-k)$ is possible in $x_{1}^{\prime}$.

Case 3: Since no $r$-move can remove a suitable rise, there exists a legal $c$-move in $x_{1}^{\prime}$. But this $c$-move has a smaller label than $(k, l)$ which is our desired contradiction.

Case 4: This case is not possible because every $c$-move has a smaller label than any $r$-move.

Combining previous two propositions, we have our first main result:

Theorem 17. The poset $P F_{n}$ is an EL-shellable poset.

\section{The order complex of $\boldsymbol{P} \boldsymbol{F}_{n}$}

In [5], it is shown that the order complex $\Delta\left(F_{n}\right)$ of fixed-point-free involutions triangulates a ball of dimension $n^{2}-n-2$. In this section we obtain a similar result for $P F_{n}$.

Lemma 18. For all $n \geqslant 2$,

$$
\operatorname{dim} \Delta\left(P F_{n}\right)=\ell\left(P F_{n}\right)=n+(n-1)+\cdots+1-n=\left(\begin{array}{l}
n \\
2
\end{array}\right) .
$$

Proof. Straightforward by using (10).

We continue by analyzing the intervals of length two.

Lemma 19. Each length two interval $[y, x] \subseteq P F_{n}$ has at most four elements.

Proof. Just as in the proof of Theorem 17, if $y<z<x$, then there are 4 cases to consider:

Case 1: type $((y, z))=c$, and type $((z, x))=c$,

Case 2: type $((y, z))=r$, and type $((z, x))=r$,

Case 3: type $((y, z))=c$, and type $((z, x))=r$,

Case 4: type $((y, z))=r$, and type $((z, x))=c$. 
In the first case, $[y, x]$ is isomorphic to an interval in $F_{m}$ for some $m \leqslant n$, and therefore, it has at most 4 elements (since $F_{m}$ is a connected subposet of $I_{m}$, which is Eulerian).

In the second case, we look at the one-line notations of $y$ and $x$. If $z$ is obtained from $y$ by setting two non-zero entries of $y$ to 0 's, and if, at the same time, $x$ is obtained from $z$ by setting two non-zero entries of $z$ to 0 's, then $y$ and $x$ differ at exactly 4 entries. Therefore, $[y, x]$ contains at most one other element other than $z$, which is obtained from $y$ by setting two entries of $y$ to 0 's. If $z$ is obtained by increasing the $i$-th entry $y_{i}$ of $y$ to $z_{i}$, and if, at the same time, $x$ is obtained from $z$ by increasing the $i$-th entry $z_{i}$ of $z$ to $x_{i}$, then $[y, x]$ has exactly 3 elements. If $z$ is obtained from $y$ by increasing the $i$-th entry $y_{i}$ of $y$ to $z_{i}$, and if $x$ is obtained from $z$ with no overlap with the replaced/increased entries of $y$, then $[y, x]$ has exactly 4 elements. Finally, if $z$ is obtained by increasing the $i$-th entry $y_{i}$ of $y$ to $z_{i}$, and $x$ is obtained from $z$ by replacing the $z_{i}$-th entry of $z$ by 0 , then $y$ and $x$ differ at exactly at 4 positions. Therefore, the interval $[y, x]$ have at most 4 elements.

Since the arguments of Case 3 and Case 4 are identical, we handle Case 3 only. Suppose that there exist more than 4 elements in $[y, x]$. Since one of the elements $y<z<x$ is obtained from $y$ by a $c$-move, the covering type of any other $y<z_{1}<x$ is not of type $c$. Otherwise, to obtain $x$ from $z$ we need to apply another $c$-move to $z$. But then the matrix ranks of $y$ and $x$ would be the same. Therefore, we conclude that if $z_{1} \neq z$ and $y<z_{1}<x$, then $z_{1}$ is obtained from $y$ by an $r$-move, and $x$ is obtained from $z_{1}$ by a $c$-move. Now it is clear that it is impossible to have another element $y<z_{2}<x$ such that $z_{2}$ covers $y$ by an $r$-move and $z_{2} \notin\left\{z, z_{1}\right\}$. Therefore $[y, x]$ have exactly 4 elements and the proof is complete.

We know from [9] that a pure, shellable simplicial complex $\Delta$ of which every $\operatorname{dim} \Delta-1$ face is contained in at most two facets is homeomorphic to either a ball, or a sphere. By Lemma 19 , we see that $\Delta\left(P F_{n}\right)$ satisfies this property.

Theorem 20. Let $\widetilde{P F}_{n}$ denote the proper part of $P F_{n}$, namely the subposet obtained from $P F_{n}$ by removing its smallest and the largest elements. For $n \geqslant 3$, the order complex $\Delta\left(\widetilde{P F}_{n}\right)$ triangulates a ball of dimension $\operatorname{dim} \Delta\left(P F_{n}\right)-2=\left(\begin{array}{l}n \\ 2\end{array}\right)-2$.

Proof. By the discussion above, it is enough to show that the reduced Euler characteristic of $\Delta\left(\widetilde{P F_{n}}\right)$ is 0 .

By Hall's Theorem (see Chapter 3, [21]), we know that the reduced Euler characteristic of an order complex of a poset $P$ is equal to the value of the Möbius function $\mu_{\widehat{P}}$ on the interval $[\hat{0}, \hat{1}]$, where $\widehat{P}$ is $P$ with a $\hat{0}$ (a smallest element) and a $\hat{1}$ (a largest element) adjoined. Therefore, it is enough to show that $\mu_{P F_{n}}([\hat{0}, \hat{1}])=0$, where $\hat{0}=(0, \ldots, 0)$ and $\hat{1}=(0, \ldots, 0, n, n-1)$.

Let $P F_{n}^{*}$ denote the dual of $P F_{n}$. By abuse of notation we use $\hat{0}$ for the smallest element of $P F_{n}^{*}$ although it is $\hat{1}$ of $P F_{n}$. Similarly, we denote the largest element of $P F_{n}^{*}$ by $\hat{1}$. Now, since $\mu_{P F_{n}}([\hat{0}, \hat{1}])=\mu_{P F_{n}^{*}}([\hat{0}, \hat{1}])$, we are going to show that the later value is zero.

It is easy to see that the cardinality of the set $\left\{x \in P F_{n}^{*}: \ell_{P F_{n}^{*}}([\hat{0}, x]) \leqslant 3\right\}$ is 1 , for $n \geqslant 3$. Indeed, if $\ell_{P F_{n}^{*}}(x)=3$, then in one-line notation $x=(0, \ldots, 0, n, 0, n-2)$, and $[\hat{0}, x]=\left\{\hat{0}<0<z_{0}<x\right\}$, where $z_{0}=(0, \ldots, 0, n, n-1)$. 
For simplicity, let us denote $\mu_{P F_{n}^{*}}$ by $\mu$, and denote the length function $\ell_{P F_{n}^{*}}$ by $\ell$. We prove by induction that $\mu([\hat{0}, z])=0$ for all $z$ with $\ell(z)>1$. Our base case is when $\ell(z)=2$. In this case, $[\hat{0}, z]$ is a chain of length 2 by the discussion in the previous paragraph, and hence, the corresponding value is 0 . Now assume that $\mu([\hat{0}, z])=0$ for all $z$ with $2 \leqslant \ell(z) \leqslant s$, and let $z^{\prime} \in P F_{n}^{*}$ be an element with $\ell\left(z^{\prime}\right)=s+1$. Since

$$
\mu\left(\left[\hat{0}, z^{\prime}\right]\right)=-\sum_{\hat{0} \leqslant z<z^{\prime}} \mu([\hat{0}, z])=-(\mu([\hat{0}, \hat{0}])+\mu([\hat{0}, 0])=-(1+(-1))=0,
$$

the proof is complete.

\section{Length-generating functions}

Recall that the standard form of an involution $\pi \in I_{n}$ is a product of transpositions of the form

$$
\pi=\left(i_{1}, j_{1}\right)\left(i_{2}, j_{2}\right) \cdots\left(i_{m}, j_{m}\right)
$$

where for all $1 \leqslant t \leqslant m, i_{t}<j_{t}$ and $i_{1}<i_{2}<\cdots<i_{m}$. We call the transpositions appearing in (12) as arcs. Using bijection (4) from the Introduction section, we identify the elements of $P F_{n}$ as involutions in $S_{n}$. With this identification, let us denote by $I(n, k)$ the set of involutions of $S_{n}$ having $k$ arcs, and define its length generating function by

$$
\mathfrak{i}_{q}(n, k):=\sum_{\pi \in I(n, k)} q^{\ell_{P F_{n}}(\pi)}
$$

Recall also that the $q$-analog of a natural number $n \in \mathbb{N}$ is the polynomial $[n]_{q}=$ $1+q+\cdots+q^{n-1}$.

Proposition 21. For all $n \geqslant 2$ and $k \in\{2, \ldots, n\}$, we have

$$
\mathfrak{i}_{q}(n+1, k)=q^{n} \mathfrak{i}_{q}(n, k)+[n]_{q} \mathfrak{i}_{q}(n-1, k-1) .
$$

Proof. We begin with defining a bijection:

$$
\Phi: I(n+1, k) \rightarrow I(n, k) \bigcup(\{2,3, \ldots, n, n+1\} \times I(n-1, k-1)) .
$$

Let $\pi$ be an element of $I(n+1, k)$. If $\pi(1)=1$, then we define $\Phi(\pi)=\sigma \in I(n, k)$ as follows: $\sigma(j)=\pi(j+1)$ for $j \in\{1,2, \ldots, n\}$. In other words, in matrix notation, $\sigma$ is obtained from $\pi$ by deleting its first row and its first column. Notice that if $\pi(1)=1$, then $\ell_{P F_{n+1}}(\pi)=\ell_{P F_{n}}(\sigma)+n$, since when we delete the first zero row from the rank-control matrix, the parameter $\rho_{<}$decreases by $n$, which is the number of zeros in this row in positions from 2 to $n+1$. 
Suppose now that $\pi(1)=i \in\{2,3, \ldots, n+1\}$. In this case, we define $\Phi(\pi)$ to be the pair $\Phi(\pi)=(i, \sigma)$, where $\sigma$ is the involution from $I(n-1, k-1)$ defined by

$$
\sigma(j)= \begin{cases}\pi(j+1) & \text { if } j \in\{1, . ., i-2\}, \\ \pi(j+2) & \text { if } j \in\{i-1, . ., n-1\} .\end{cases}
$$

In matrix notation, $\sigma$ is obtained from $\pi$ by deleting the first and the $i$-th rows of $\pi$, as well as deleting its first and $i$-th columns. In this case we have: $\ell_{P F_{n+1}}(\pi)=\ell_{P F_{n-1}}(\sigma)+i-2$. To see this, notice that all the equalities in the upper triangular portion of $R k(\pi)$ are carried into that of $R k(\sigma)$ with additional $i-2$ equalities arising from the 0's at the positions $(1,2),(1,3), \ldots,(1, i-1)$ of $\pi$. Thus $\rho_{<}(\pi)=\rho_{<}(\sigma)+i-1$. On the other hand, since the ranks of $\pi$ and $\sigma$ differ by 2 , and their sizes differ by 2 , by the formula (10), we see that

$$
\begin{aligned}
\ell_{P F_{n+1}}(\pi) & =\rho_{<}(\pi)-\frac{2(n+1)-r k(\pi)}{2} \\
& =\rho_{<}(\sigma)+i-1-\frac{2(n-1)-r k(\sigma)+2}{2} \\
& =\ell_{P F_{n-1}}(\sigma)+i-2 .
\end{aligned}
$$

See Example 22 for an illustration.

Now, in the light of these observations, we derive the desired recurrence:

$$
\begin{aligned}
\mathfrak{i}_{q}(n+1, k) & =\sum_{\pi \in I(n+1, k)} q^{\ell_{P F_{n+1}}(\pi)} \\
& =\sum_{\pi \in I(n+1, k), \pi(1)=1} q^{\ell_{P F_{n}+1}(\pi)}+\sum_{\pi \in I(n+1, k), \pi(1) \neq 1} q^{\ell_{P F_{n+1}}(\pi)} \\
& =\sum_{\sigma \in I(n, k)} q^{\ell_{P F_{n}}(\sigma)+n}+\sum_{i=2}^{n+1} \sum_{\sigma \in I(n-1, k-1)} q^{\ell_{P F_{n-1}}(\sigma)+i-2} \\
& =q^{n} \cdot \sum_{\sigma \in I(n, k)} q^{\ell_{P F_{n}}(\sigma)}+\sum_{i=2}^{n+1} q^{i-2} \cdot \sum_{\sigma \in I(n-1, k-1)} q^{\ell_{P F_{n-1}}(\sigma)} \\
& =q^{n_{1}} \mathfrak{i}_{q}(n, k)+\left(1+q+q^{2}+\cdots+q^{n-1}\right) \mathfrak{i}_{q}(n-1, k-1) \\
& =q^{n_{1}}(n, k)+[n]_{q} \mathfrak{i}_{q}(n-1, k-1) .
\end{aligned}
$$

Example 22. Let us consider an example in order to understand (13). Consider

$$
\pi=\left(\begin{array}{llllll}
0 & 0 & 0 & 0 & 1 & 0 \\
0 & 0 & 0 & 0 & 0 & 0 \\
0 & 0 & 0 & 0 & 0 & 0 \\
0 & 0 & 0 & 0 & 0 & 1 \\
1 & 0 & 0 & 0 & 0 & 0 \\
0 & 0 & 0 & 1 & 0 & 0
\end{array}\right) \text { with } R k(\pi)=\left(\begin{array}{llllll}
0 & 0 & 0 & 0 & 1 & 1 \\
0 & 0 & 0 & 0 & 1 & 1 \\
0 & 0 & 0 & 0 & 1 & 1 \\
0 & 0 & 0 & 0 & 1 & 2 \\
1 & 1 & 1 & 1 & 2 & 3 \\
1 & 1 & 1 & 2 & 3 & 4
\end{array}\right)
$$




$$
\sigma=\Phi(\pi)=\left(\begin{array}{llll}
0 & 0 & 0 & 0 \\
0 & 0 & 0 & 0 \\
0 & 0 & 0 & 1 \\
0 & 0 & 1 & 0
\end{array}\right) \text { with } R k(\sigma)=\left(\begin{array}{llll}
0 & 0 & 0 & 0 \\
0 & 0 & 0 & 0 \\
0 & 0 & 0 & 1 \\
0 & 0 & 1 & 2
\end{array}\right) .
$$

By using (10), it is easy to verify $\ell_{P F_{6}}(\pi)=12-4=8$ and $\ell_{P F_{4}}(\sigma)=8-3=5$.

\subsection{An explicit formula for $\mathfrak{i}_{q}(n, k)$.}

Let $\pi \in P F_{n}$ be a partial involution and let $\pi=\left(i_{1}, j_{1}\right)\left(i_{2}, j_{2}\right) \cdots\left(i_{m}, j_{m}\right)$ denote its standard form viewed as an involution in $I_{n}$ via bijection (4). It follows from the proof of Proposition 6.2 of [8] that the following equality is true:

$$
\ell_{P F_{n}}(\pi)=\rho_{<}(\pi)=\widetilde{i n v}(\pi)+\sum_{a: \pi(a)=a}(n-a)
$$

where $\widetilde{i n v}(\pi)$ is the "modified inversion number," which is equal to the number of inversions in the word $i_{1} j_{1} i_{2} j_{2} \cdots i_{m} j_{m}$.

Proposition 23. $\mathfrak{i}_{q}(2 k, k)=[2 k-1]_{q} !$ !.

Proof. By Proposition 21 we have

$$
\mathfrak{i}_{q}(2 k, k)=q^{2 n-1} \mathfrak{i}_{q}(2 k-1, k)+[2 k-1]_{q} \mathfrak{i}_{q}(2 k-2, k-1) .
$$

Since there are no involutions in $S_{2 k-1}$ which have $k$ arcs (the maximal number of arcs for an involution in $S_{2 k-1}$ is $\left.k-1\right)$, we have $\mathfrak{i}_{q}(2 k-1, k)=0$ and therefore $\mathfrak{i}_{q}(2 k, k)=$ $[2 k-1]_{q} \mathfrak{i}_{q}(2 k-2, k-1)=[2 k-1]_{q} \mathfrak{i}_{q}(2(k-1), k-1)$. Now, by induction we get $\mathfrak{i}_{q}(2 k, k)=$ $[2 k-1]_{q} ! !$

\section{Proposition 24.}

$$
\mathfrak{i}_{q}(n, k)=q^{\left(\begin{array}{c}
n-2 k \\
2
\end{array}\right)} \cdot\left(\begin{array}{c}
n \\
2 k
\end{array}\right)_{q} \cdot[2 k-1]_{q} ! !
$$

where $\left(\begin{array}{c}n \\ 2 k\end{array}\right)_{q}=\frac{[n]_{q} !}{[2 k]_{q} ![n-2 k]_{q} !}$.

Proof. Let $\pi$ an element from $I(n, k)$. The involution $\pi \in S_{n}$ has $k$ arcs, hence, it has $n-2 k$ fixed points. Thus, $n-2 k$ zero rows and columns in the corresponding partial fixed-point-free involution matrix. So, there is a natural bijection

$$
\pi \leftrightarrow\left(\left\{i_{1}, \ldots, i_{n-2 k}\right\}, \sigma\right)
$$

where $1 \leqslant i_{1}<i_{2}<\cdots<i_{n-2 k} \leqslant n$ are the fixed points of $\pi$ and $\sigma \in I(2 k, k)$ is the fixed point free involution of $S_{2 k}$, whose partial fixed-point-free involution matrix is obtained from $\pi$ by deleting zero rows and columns. Now, using formula (14) we have

$$
\mathfrak{i}_{q}(n, k)=\sum_{\pi \in I(n, k)} q^{\ell_{P F_{n}}(\pi)}
$$




$$
\begin{aligned}
& =\sum_{\substack{\left(\left\{i_{1}, \ldots, i_{n-2 k}\right\} \\
\sigma\right):}} q^{n-i_{1}+n-i_{2}+\cdots+n-i_{n-2 k}+\ell_{F_{2 k}}(\sigma)} \\
& =\left(\sum_{1 \leqslant i_{1}<i_{2}<\cdots<i_{n-2 k} \leqslant n} q^{n-i_{1}+\cdots+n-i_{n-2 k}}\right) \cdot\left(\sum_{\sigma \in I(2 k, k)} q^{\ell_{\ell_{2 k}}(\sigma)}\right) \\
& =\left(\sum_{0 \leqslant j_{1}<\cdots<j_{n-2 k} \leqslant n-1} q^{j_{1}+\cdots+j_{n-2 k}}\right) \cdot \mathfrak{i}_{q}(2 k, k) .
\end{aligned}
$$

To simplify (15), we use well known Gaussian identity (see [21], formula (1.87)):

$$
\prod_{i=0}^{j-1}\left(1+x q^{i}\right)=\sum_{k=0}^{j} x^{k} q^{\left(\begin{array}{c}
k \\
2
\end{array}\right)}\left(\begin{array}{l}
j \\
k
\end{array}\right)_{q}
$$

which is equivalent, by expanding the product, to

$$
\sum_{0 \leqslant s_{1}<s_{2}<\cdots<s_{k} \leqslant j-1} q^{\sum_{r=1}^{k} s_{r}} x^{k}=\sum_{k=0}^{j} x^{k} q^{\left(\begin{array}{c}
k \\
2
\end{array}\right)}\left(\begin{array}{l}
j \\
k
\end{array}\right)_{q}
$$

Replacing $j$ by $n$, and comparing the coefficients of $x^{n-2 k}$ in (17), we obtain our desired formula

$$
\mathfrak{i}_{q}(n, k)=q^{\left(\begin{array}{c}
n-2 k \\
2
\end{array}\right)} \cdot\left(\begin{array}{c}
n \\
2 k
\end{array}\right)_{q} \cdot[2 k-1]_{q} ! !
$$

\subsection{Length generating function of $P F_{n}$}

Next, we look at the length generating function of $P F_{n}$ more closely.

$$
\mathfrak{p}_{q}(n):=\sum_{\pi \in P F_{n}} q^{\ell_{P F_{n}}(\pi)}=\sum_{k=0}^{\left\lfloor\frac{n}{2}\right\rfloor} \mathfrak{i}_{q}(n, k) .
$$

By a straightforward calculation we see that $\mathfrak{p}_{q}(1)=1, \mathfrak{p}_{q}(2)=1+q, \mathfrak{p}_{q}(3)=1+q+q^{2}+q^{3}$.

Proposition 25. For all $n \geqslant 2$, we have

$$
\mathfrak{p}_{q}(n+1)=q^{n} \mathfrak{p}_{q}(n)+[n]_{q} \mathfrak{p}_{q}(n-1) .
$$

Proof. Follows from Proposition 21.

Example 26. It is easy to verify the following calculation from the Hasse diagram of $P F_{4}$ in Figure 2: $\mathfrak{p}_{q}(4)=q^{3} \mathfrak{p}_{q}(3)+[3]_{q} \mathfrak{p}_{q}(2)=1+2 q+2 q^{2}+2 q^{3}+q^{4}+q^{5}+q^{6}$. 


\subsection{Skew-symmetric matrices over $\mathbb{F}_{q}$}

There is an interesting similarity between the rank generating function $\mathfrak{i}_{q}(n, k)$ and the number of $\mathbb{F}_{q}$-rational points of rank $2 k, n \times n$ skew symmetric matrices, which we denote by Skew ${ }_{n}^{2 k}$. Here $\mathbb{F}_{q}$ is the finite field with $q$ elements. It is well known that the number of $\mathbb{F}_{q}$-rational points of the general linear group $\mathrm{GL}_{n}$ and the symplectic group $\operatorname{Sp}_{n}(n=2 m)$ are given by

$$
\left|\mathrm{GL}_{n}\right|_{\mathbb{F}_{q}}=q^{\left(\begin{array}{c}
n \\
2
\end{array}\right)} \prod_{i=1}^{n}\left(q^{i}-1\right) \text { and }\left|\mathrm{Sp}_{2 m}\right|_{\mathbb{F}_{q}}=q^{m^{2}} \prod_{i=1}^{m}\left(q^{2 i}-1\right) .
$$

The group $G=\mathrm{GL}_{n}$ acts $\mathrm{Skew}_{n}^{2 k}$ transitively. A simple matrix computation shows that

$$
\left|G_{x}\right|_{\mathbb{F}_{q}}=\left|\mathrm{GL}_{n-2 k}\right|_{\mathbb{F}_{q}}\left|\mathrm{Sp}_{2 k}\right|_{\mathbb{F}_{q}}\left|\mathrm{Mat}_{n-2 k, 2 k}\right|_{\mathbb{F}_{q}}
$$

where $\mathrm{Mat}_{n-2 k, 2 k}$ is the space of $2 k \times(n-2 k)$ matrices. Thus,

$$
\left|\operatorname{Skew}_{n}^{2 k}\right|_{\mathbb{F}_{q}}=\left|G / G_{x}\right|_{\mathbb{F}_{q}}=\frac{q^{\left(\begin{array}{c}
n \\
2
\end{array}\right)} \prod_{i=1}^{n}\left(q^{i}-1\right)}{q^{k^{2}} \prod_{i=1}^{k}\left(q^{2 i}-1\right) q^{\left(\begin{array}{c}
n-2 k \\
2
\end{array}\right)} \prod_{i=1}^{n-2 k}\left(q^{i}-1\right) q^{2 k(n-2 k)}},
$$

which simplifies as follows

$$
\begin{aligned}
\left|\operatorname{Skew}_{n}^{2 k}\right|_{\mathbb{F}_{q}} & =q^{\left(\begin{array}{c}
n \\
2
\end{array}\right)-k^{2}-\left(\begin{array}{c}
n-2 k \\
2
\end{array}\right)-2 k(n-2 k)} \frac{[n] !(q-1)^{n}}{\left(\prod_{i=1}^{k}[2 i]\right)(q-1)^{k}[n-2 k] !(q-1)^{n-2 k}} \\
& =q^{2\left(\begin{array}{c}
k \\
2
\end{array}\right)} \frac{[n] !(q-1)^{k}}{\left(\prod_{i=1}^{k}[2 i]\right)[n-2 k] !} \\
& =q^{2\left(\begin{array}{c}
k \\
2
\end{array}\right)}(q-1)^{k}\left(\begin{array}{c}
n \\
2 k
\end{array}\right)_{q}[2 k-1] ! ! .
\end{aligned}
$$

In other words, $\left|\operatorname{Skew}_{n}^{2 k}\right|_{\mathbb{F}_{q}}=\mathfrak{i}_{q}(n, k) q^{2\left(\begin{array}{c}k \\ 2\end{array}\right)-\left(\begin{array}{c}n-2 k \\ 2\end{array}\right)}(q-1)^{k}$.

\section{References}

[1] Eli Bagno, Yonah Cherniavsky. Congruence $B$-orbits and the Bruhat poset of involutions of the symmetric group. Discrete Math., 6(312):1289-1299, 2012.

[2] Anders Björner. Shellable and Cohen-Macaulay partially ordered sets. Trans. Amer. Math. Soc., 1(260):159-183, 1980.

[3] Anders Björner, Michelle Wachs. Bruhat order of Coxeter groups and shellability. Advan. in Math., 1(43):87-100, 1982.

[4] Mahir Bilen Can. The rook monoid is lexicographically shellable. Adv. Applied Math., to appear. arXiv:1001.5104.

[5] Mahir Bilen Can, Yonah Cherniavsky, and Tim Twelbeck. Lexicographic shellability of the Bruhat-Chevalley order on fixed-point-free involutions. Israel J. Math., to appear. arXiv:1211.4147. 
[6] Mahir Bilen Can and Lex Renner. Bruhat-Chevalley order on the rook monoid. Turkish J. Math., 2(35):1-21, 2011.

[7] Mahir Bilen Can, Tim Twelbeck. Lexicographic shellability of partial involutions. Discrete Math. , (335):66-80, 2014.

[8] Yonah Cherniavsky. On involutions of the symmetric group and congruence $B$-orbits of anti-symmetric matrices. Internat. J. Algebra Comput., 5(21):841-856, 2011.

[9] Gopal Danaraj, Victor Klee. Shellings of spheres and polytopes. Duke Math. J., (41):443-451, 1974.

[10] Matthew J. Dyer. Hecke algebras and shellings of Bruhat intervals. Compositio Math., 1(89):91-115, 1993.

[11] Paul H. Edelman. The Bruhat order of the symmetric group is lexicographically shellable. Proc. Amer. Math. Soc., 3(82):355-358, 1981.

[12] Axel Hultman. Fixed points of involutive automorphisms of the Bruhat order. Adv. Math., 1(195):283-296, 2005.

[13] Axel Hultman. Twisted identities in Coxeter groups. J. Algebraic Combin., 2(28):313-332, 2008.

[14] Federico Incitti. The Bruhat order on the involutions of the symmetric group. $J$. Algebraic Combin., 3(20):243-261, 2004.

[15] Federico Incitti. Bruhat order on the involutions of classical Weyl groups. Adv. in Appl. Math., 1(37):68-111, 2006.

[16] Robert A. Proctor. Classical Bruhat orders and lexicographic shellability. J. Algebra, 1(77):104-126, 1982.

[17] Mohan Putcha. Shellability in reductive monoids. Trans. Amer. Math. Soc., 1(354):413-426, 2002.

[18] Lex Renner. Analogue of the Bruhat decomposition for algebraic monoids. J Algebra, 2(101):303-338, 1986.

[19] R.W. Richardson, T.A. Springer. The Bruhat order on symmetric varieties. Geom. Dedicata, 1-3(35):389-436, 1990.

[20] R.W. Richardson, T.A. Springer. Complements to: 'The Bruhat order on symmetric varieties'. Geom. Dedicata, 2(49):231-238, 1994.

[21] Richard Stanley. Enumerative Combinatorics. Volume 1. Second Edt. Cambridge Studies in Advanced Mathematics, Volume 49. Cambridge University Press. 2012.

[22] Fernando Szechtman. Equivalence and congruence of matrices under the action of standard parabolic subgroups. Electron. J. Linear Algebra, (16):325-333, 2007.

[23] Michelle Wachs. Poset topology: tools and applications. Geometric Combinatorics, IAS/Park City Math. Ser., Volume 13, 497-615, Amer. Math. Soc. 2007.

[24] Lauren Williams. Shelling totally nonnegative flag varieties. J. Reine Angew. Math., (609):1-21, 2007. 